\title{
EGFR NP_005219.2:p.V774_C775insHV
}

National Cancer Institute

\section{Source}

National Cancer Institute. EGFR NP 005219.2:p.V774 C775insHV. NCI Thesaurus. Code C98695.

An insertion of the amino acid sequence histidine-valine between the valine at position 774 and the cysteine at position 775 of the epidermal growth factor receptor protein. 